\title{
Surgical Ligation of Patent Ductus Arteriosus Using the Descending Aortic Approach in Two Dogs
}

\author{
Dae-Hyun Kim****1, Sung-Hwa Hong*, Hyunwook Myung*, Dong-ju Son*, Aryung Nam*, \\ Sung-Yong Jung*, Jung-Yeon Hwang* and Hyun-Choul Jee* \\ *Helix Animal Medical Center, Seoul 05581, Korea \\ **Department of Medical Engineering, Yonsei University College of Medicine, Seoul 03722, Korea
}

(Received: October 07, 2019 / Accepted: January 28, 2020)

\begin{abstract}
Surgical ligation is the treatment of the choice in patients with patent ductus arteriosus (PDA). This case series presents two cases of PDA, one with and one without persistent left cranial vena cava (PLCVC), treated with surgical ligation through the descending aortic approach with mini-thoracotomy. There were no specific complications during the surgical procedures. The descending aortic approach would be an alternative method for dissection of the PDA.
\end{abstract}

Key words : patent ductus arteriosus, descending aortic approach, dorsolateral mini-thoracotomy, dog.

\section{Introduction}

Patent ductus arteriosus (PDA) is one of the most common congenital heart diseases in dogs; it is characterized by the abnormal persistence of the shunt between the aorta and the pulmonary artery $(2,8)$. Physiologically, the PDA causes left to right shunting and results in volume overload to the left side of the heart (8). Consequently, the left heart is enlarged and finally, in many cases, left heart failure occurs (2). According to a previous report, if PDA is left untreated, around $70 \%$ of the affected dogs could die of left heart failure in the first 18 months of life (8).

There are two main treatment methods: non-surgical and surgical (5). The non-surgical method involves interventional cardiology and is based on catheters and thrombo-emboligenic devices $(5,10)$. This method is relatively non-invasive; however, it is difficult to apply in dogs weighing less than $2.5 \mathrm{~kg}$ because their peripheral arteries are too small to place a vascular sheath (11). In such patients, surgical ligation is acceptable as the treatment of choice (11). Traditionally, the ductus arteriosus is approached dorsally by elevating the vagus nerve, which is used as a landmark for PDA surgery $(2,5,8,12,15)$. However, there is a risk of catastrophic intraoperative hemorrhage if the medial wall of the ductus arteriosus is weak $(2,6)$.

Occasionally, a persistent left cranial vena cava (PLCVC) will exist in addition to the PDA $(7,14)$. It is recommended that the PLCVC should not be ligated or dissected (8). As the PLCVC anatomically overlies the ductus arteriosus, it should be carefully separated and retracted dorsally along with the vagus nerve in order to proceed with the dissection of the ductus arteriosus $(7,8)$.

The purpose of this report was to introduce the descending

${ }^{1}$ Corresponding author.

E-mail : vet1982@naver.com aortic approach as a safer alternative for the dissection of the PDA as it requires a smaller incision compared to the standard technique and does not involve manipulation of the PLCVC.

\section{Case Report}

\section{Case 1}

A 5-year-old female Maltese dog weighing $3.2 \mathrm{~kg}$ was referred to the Hae-deun Animal Medical Center for PDA surgery with a history of previous PDA coiling failure. The patient was on regular oral medication $(0.2 \mathrm{mg} / \mathrm{kg}$ pimobendan, $1.2 \mathrm{mg} / \mathrm{kg}$ furosemide, $1.3 \mathrm{mg} / \mathrm{kg}$ sildenafil, and 0.125 $\mathrm{mg} / \mathrm{kg}$ ramipril twice daily). On physical examination, a grade $\mathrm{V}$ continuous murmur with a palpable thrill was detected. Systolic blood pressure was estimated to be $\sim 180 \mathrm{mmHg}$. Other animal conditions like body temperature, and hematology were normal. Radiological findings showed severe cardiomegaly and enlargement of the left atrium and ventricle, the coil from the previous procedure was also observed at the level of the right $8^{\text {th }}$ rib (Fig 1A). The PDA was also confirmed using color flow Doppler echocardiography (Aplio 300 ultrasound system — Toshiba Medical Systems; Tokyo, Japan) and computed tomography (CT) (BrightSpeed Elite 16 Slice CT — GE Healthcare; Chicago, Illinois, United States) (Fig 1B). In preparation for the surgery, the animal was premedicated intravenously with $0.2 \mathrm{mg} / \mathrm{kg}$ butorphanol (Butophan inj., Myungmoon Pharm, Co., Ltd.) and $0.3 \mathrm{mg} /$ kg midazolam (Midazolam inj., Bukwang Pharm, Co., Ltd.). $22 \mathrm{mg} / \mathrm{kg}$ of cefazolin (Cefazolin inj., Chongkundang) was given intravenously as a precaution. Anesthesia was induced intravenously using $2 \mathrm{mg} / \mathrm{kg}$ etomidate (Etomidate-lipuro inj., B-BRAUN Korea) and maintained with $2.0 \%$ isoflurane inhalation. With the patient placed in a right lateral position and the surgeon standing at the patient's back, a $3 \mathrm{~cm}$ long dorsolateral incision was made, followed by a left $4^{\text {th }}$ thora- 

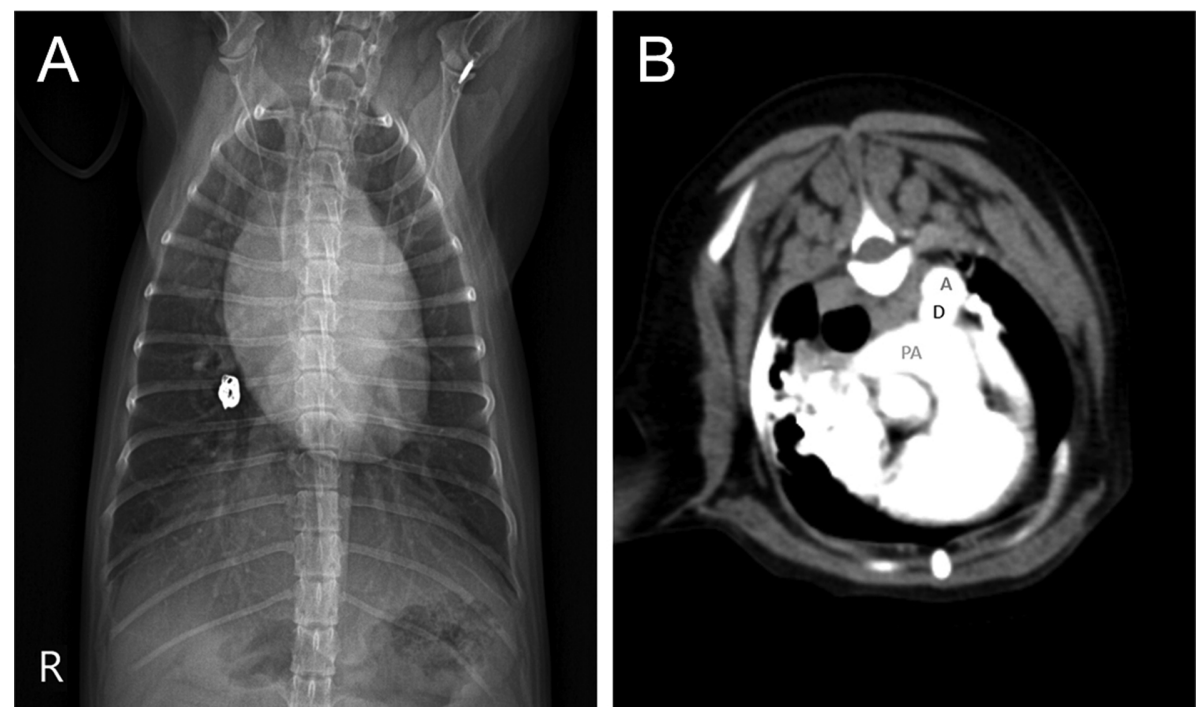

Fig 1. (A) Thoracic radiography of case \#1 shows an enlarged left atrium and left ventricle. The thrombo-emboligenic coil is seen in the right branch of the pulmonary artery. (B) The patent ductus arteriosus is clearly seen in the computed tomography imaging of case \#1 (A: aorta; D: ductus arteriosus; PA: pulmonary artery).

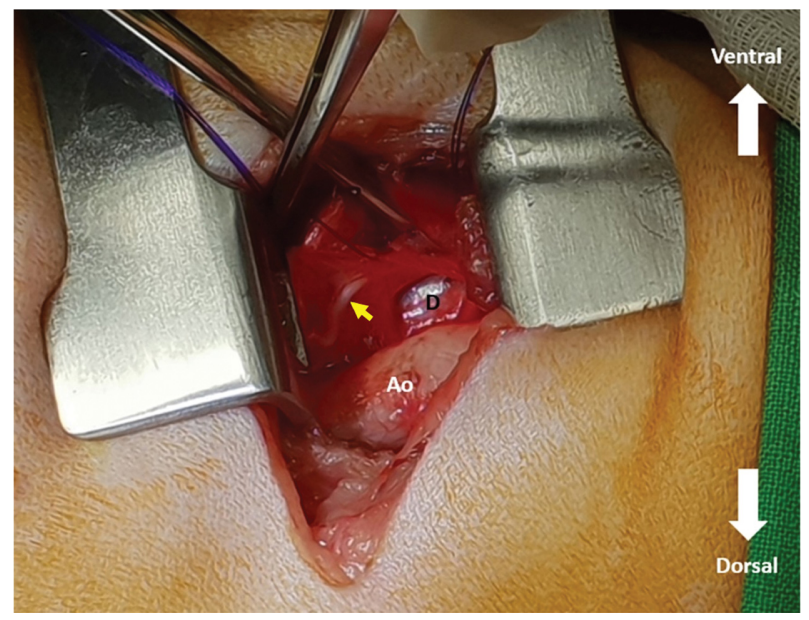

Fig 2. The mediastinal pleura is elevated with two stay sutures. The vagus nerve (yellow arrow), ductus arteriosus (D), and the aorta (Ao) can be seen in the image.

cotomy. The mediastinal pleura was incised over the aorta and reflected ventrally using two 4-0 polyprolene (Surgipro ${ }^{\mathrm{TM}} \mathrm{II}$, Covidien $^{\mathrm{TM}}$ ) stay sutures (Fig 2). A relatively short PDA was confirmed visually and carefully dissected, a rubber tourniquet was then placed with the help of 1-0 black silk (BLACK SILK, Ailee Co., Ltd.). Considering the patient's age, the team temporarily occluded the shunting vessel with the tourniquet for about $10 \mathrm{~min}$, to check if the heart would adapt after PDA closure. After confirming that the cardiac rhythm and blood pressure were well maintained, the shunting vessel was finally ligated. The murmur and thrill revealed on initial examination were found to have disappeared following the surgery. To support left ventricular function, oral medication was continued for 3 months after surgery.

\section{Case 2}

A 6-month-old male Pomeranian dog weighing $1.8 \mathrm{~kg}$ was referred to the Helix Animal Medical Center for PDA sur-
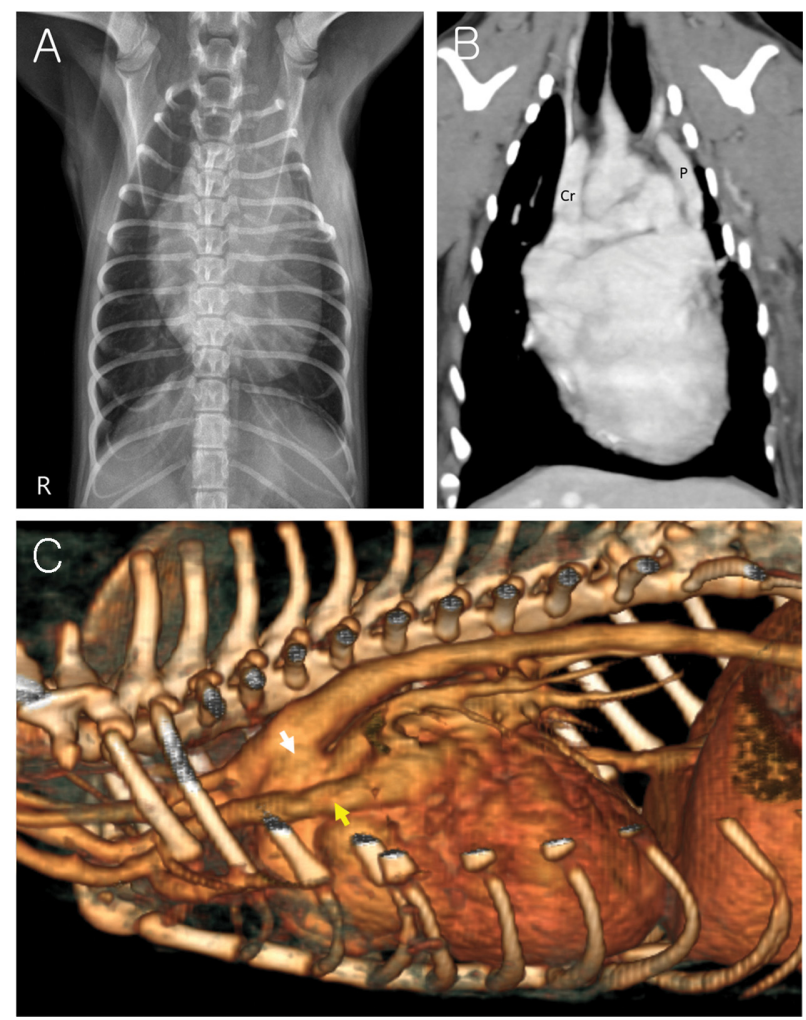

Fig 3. (A) Thoracic radiography of case \#2 revealed marked cardiomegaly. (B) Both the normal cranial vena cava $(\mathrm{Cr})$ and the persistent left cranial vena cava $(\mathrm{P})$ were identified on computed tomography. (C) A 3 dimensional volume rendering image shows the ductus arteriosus (white arrow) and the persistent left cranial vena cava (yellow arrow).

gery. 10 days ago, the patient had about $4 \mathrm{~cm}$ of thoracotomy at local animal hospital for PDA ligation, but further surgery was not performed because the abnormal vein was identified. Physical examination revealed a grade V continuous murmur with a palpable thrill. Other animal conditions 

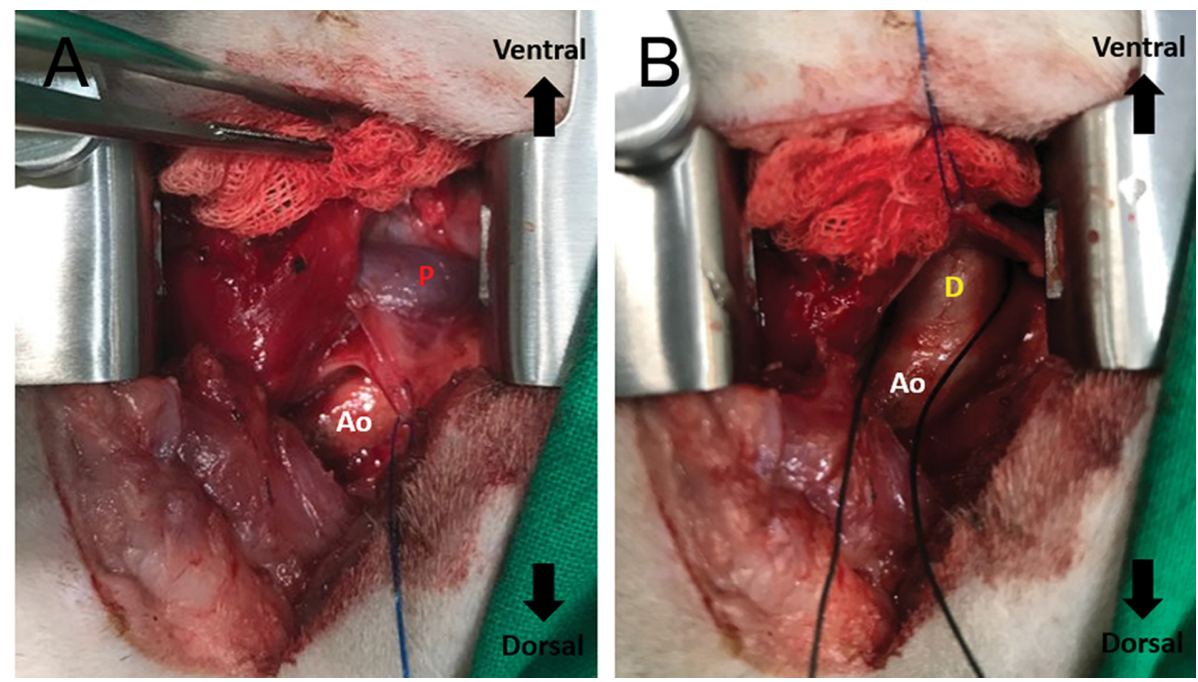

Fig 4. (A) A stay suture is placed on the mediastinal pleura. The aorta (Ao) and the persistent left cranial vena cava (P) can be seen. (B) The large patent ductus arteriosus (D) is well visualized when the mediastinal pleura is retracted.

like body temperature, blood pressure, and hematology were normal. Radiological imaging showed severe cardiomegaly, bulging of the main pulmonary artery, and enlargement of the left atrium and ventricle (Fig 3A). Echocardiography detected an increased left atrium: aorta (LA:AO) ratio (1:1.65), enlarged left atrium and ventricle, and decreased contractility in Bmode and M-mode (Aplio 300 ultrasound system — Toshiba Medical Systems; Tokyo, Japan). Furthermore, turbulent highvelocity flow was visible on color doppler examination, directed from the ductus towards the main pulmonary trunk. The diagnosis of PDA with PLCVC was confirmed using CT (Brivo 38516 Slice CT - GE Healthcare; Chicago, Illinois, United States) (Fig 3B and C). The animal was premedicated intravenously with $0.2 \mathrm{mg} / \mathrm{kg}$ butorphanol (Butophan inj., Myungmoon Pharm, Co., Ltd.) and $0.3 \mathrm{mg} / \mathrm{kg}$ midazolam (Midazolam inj., Bukwang Pharm, Co., Ltd.). $22 \mathrm{mg} / \mathrm{kg}$ cefazolin (Cefazolin inj., Chongkundang) was given intravenously as a precaution. Anesthesia was induced intravenously using $2 \mathrm{mg} / \mathrm{kg}$ etomidate (Etomidate-lipuro inj., B-BRAUN Korea) and maintained with $2.0 \%$ isoflurane inhalation. Considering the adhesion of the lung with parietal pleura, we decided to make a sufficient incision. With the patient placed in a right lateral position and the surgeon standing at the patient's back, a left $4^{\text {th }}$ thoracotomy was performed along the previous incision site. The mediastinal pleura was incised over the aorta and reflected ventrally with a 4-0 polyprolene (Surgipro $^{\mathrm{TM}} \mathrm{II}$, Covidien ${ }^{\mathrm{TM}}$ ) stay suture. Manipulation of the PLCVC was not necessary for this procedure (Fig 4A). The PDA was carefully dissected and ligated with 1-0 black silk (BLACK SILK, Ailee Co., Ltd.) (Fig 4B). The initially detected heart murmur and thrill disappeared following the surgery. No residual flow was detected by color flow Doppler echocardiography. The patient was monitored using radiography for 3 months after the surgery until the cardiomegaly was completely reversed.

\section{Discussion}

The cases presented in this article were treated with surgi- cal ligation of the PDA, with or without PLCVC, via the descending aortic approach with a mini-thoracotomy incision. There were no specific complications arising during this surgical procedure, such as hemorrhage from the ductus arteriosus or Branham's reflex.

Traumatic injury during dissection of the ductus arteriosus is one of the most serious complications of the procedure $(2,6)$. According to a retrospective study of 201 dogs treated with surgical ligation of PDA, the incidence of intraoperative hemorrhage is around $9.5 \%$ (1). To decrease this risk, various technical variations have been suggested including the Jackson-Henderson technique, hemostatic clip ligation, and a wire loop technique $(3,4,12)$. The Jackson-Henderson technique has an advantage in that it does not involve the dissection of the medial aspect of the ductus arteriosus, which is a potentially weak structure (2). However, intraoperative hemorrhage was reported to be around 8.3-12.5\% even with this method $(12,15)$. In addition, one study reported that around 53\% of PDA cases had residual flow if Jackson-Henderson technique is used (2). Using hemostatic clip ligation, intra-operative hemorrhage is reported to occur in $10 \%$ of the cases (3). Although one study of 6 cases reported no intraoperative hemorrhage using the wire loop technique, this needs to be confirmed after analyzing more cases because this technique is very similar to the traditional method, with the only difference being that a wire is passed under the ductus arteriosus (4).

Almost all the sources in veterinary literature state that the vagus nerve should be dorsally elevated to expose the ductus arteriosus $(2,5,8,12,15)$. However, it is now recommended that the mediastinal pleura be opened along the descending aorta and retracted anteriorly for general PDA surgery in human medicine (13). According to our experiences, this surgical technique has no specific limitations in applying to animals. This approach allows the immediate identification of the ductus arteriosus as well as its connection point with the aorta; this allows the surgeon to dissect the ductus arteriosus near the aorta instead of the main pulmonary trunk, which is more vulnerable (13). Additionally, dissection of the cranial, 
caudal, and medial aspects of the ductus arteriosus can be minimal; in our cases, just a puncture of the medial connective tissue with a jaws-closed, right-angle forceps was sufficient. We believe that this is due to the removal of connective tissue during the dissection of the mediastinal pleural. Another advantage of this approach is that it allows easy identification of the aortic arch, the left subclavian artery, and the brachiocephlic trunk with a simple elongation of the mediastinal pleural incision; this is helpful in preventing the inadvertent ligation of the wrong structure (13). Furthermore, this surgery can be performed with a relatively small incision (compared to the traditional ligation method) because only the aorta and the ductus arteriosus need to be identified.

PLCVC is an uncommon congenital vascular anomaly; it occurs when the left cranial cardinal veins do not atrophy and are abnormally persistent (7). Despite being a rare condition, PLCVC often co-exists with other congenital vascular anomalies including PDA $(7,9,14,16)$. Generally, ligation of the PLCVC is not recommended if it itself is not symptomatic (8). Because the PLCVC overlies the ductus arteriosus in close association, it should first be carefully dissected in order to expose the ductus arteriosus in the traditional approach $(7,8,14)$. Although associated complications have not been reported yet, this is a delicate procedure requiring meticulous dissection so as to avoid the damage to the PLCVC, as well as the ductus arteriosus and the left main pulmonary artery. In our case, however, PLCVC dissection was not necessary to expose the ductus arteriosus, as it is located outside the mediastinal pleura. Therefore, the descending aortic approach would be easier and safer in cases of PDA with PLCVC.

\section{Conclusions}

In conclusion, we successfully performed surgical ligation in two cases of PDA, with and without PLCVC, using the descending aortic approach with mini-thoracotomy. This approach is hypothesized to be safer compared to the traditional approach for surgical ligation of PDA. The major limitation of this study is that it analyzed only two cases and further studies are necessary to verify the safety and associated surgical complications of this approach using larger sample sizes.

\section{Acknowledgements}

We would like to thank the Hae-deun Animal Medical Center for providing the medical records and the permission to use their patients' information for this report.

\section{References}

1. Birchard SJ, Bonagura JD, Fingland RB. Results of ligation of patent ductus arteriosus in dogs: 201 cases (1969-1988). J Am Vet Med Assoc 1990; 196: 2011-2013.

2. Broaddus KD, Tillson DM. Patent ductus arteriosus in dogs. Compend Contin Educ Vet 2010; 32: E3.

3. Corti LB, Merkley D, Nelson OL, Ware WA. Retrospective evaluation of occlusion of patent ductus arteriosus with hemoclips in 20 dogs. J Am Anim Hosp Assoc 2000; 36: 548555.

4. Downs MO, Stampley AR, Rawlings CA. A wire loop technique for ligation of patent ductus arteriosus. J Small Anim Pract 1995; 36: 489-491.

5. Goodrich KR, Kyles AE, Kass PH, Campbell F. Retrospective comparison of surgical ligation and transarterial catheter occlusion for treatment of patent ductus arteriosus in two hundred and four dogs (1993-2003). Vet Surg 2007; 36: 4349.

6. Hunt GB, Simpson DJ, Beck JA, Goldsmid SE, Lawrence D, Pearson MR, Bellenger CR. Intraoperative hemorrhage during patent ductus arteriosus ligation in dogs. Vet Surg 2001; 30: 58-63.

7. Hwang Y, Oh H, Chang D, Kim G. Persistent left cranial vena cava with congenital heart defect in two dogs. Korean J Vet Res 2016; 56: 193-195.

8. Johnston SA, Tobias KM. Veterinary Surgery: Small Animal, 2nd Edition. Elsevier 2018: 4778-4782.

9. Larcher T, Abadie J, Roux FA, Deschamps JY, Wyers M. Persistent left cranial vena cava causing oesophageal obstruction and consequent megaoesophagus in a dog. J Comp Pathol 2006; 135: 150-152.

10. Saunders AB, Gordon SG, Boggess MM, Miller MW. Longterm outcome in dogs with patent ductus arteriosus: 520 cases (1994-2009). J Vet Intern Med 2014; 28: 401-410.

11. Selmic LE, Nelson DA, Saunders AB, Hobson HP, Saunders WB. An intrapericardial technique for PDA ligation: surgical description and clinical outcome in 35 dogs. J Am Anim Hosp Assoc 2013; 49: 31-40.

12. Stanley BJ, Luis-Fuentes V, Darke PG. Comparison of the incidence of residual shunting between two surgical techniques used for ligation of patent ductus arteriosus in the dog. Vet Surg 2003; 32: 231-237.

13. Susheel Kumar TK. Surgical management of patent ductus arteriosus. Congenit Heart Dis 2019; 14: 57-59.

14. Uemura A, Tanaka R. Surgical closure of patent ductus arteriosus with persistent left cranial vena cava in an infant dog. HVM Bioflux 2017; 9: 106-109.

15. Van Israël N, French AT, Dukes-McEwan J, Corcoran BM. Review of left-to-right shunting patent ductus arteriosus and short term outcome in 98 dogs. J Small Anim Pract 2002; 43: 395-400.

16. Zani A, Becchetti E, Leonardi P, Sinatra A. Persistent left cranial vena cava draining into the left atrium associated with pulmonary stenosis in a French bulldog. J Vet Cardiol 2014; 16: $121-125$ 